\title{
Dynamic thiol/disulphide homeostasis in acute pancreatitis
}

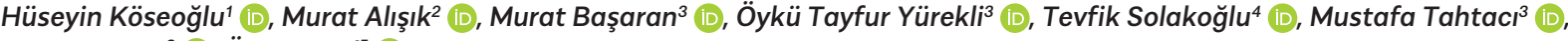 \\ Osman Ersoy ${ }^{3}$ (D), Özcan Erel ${ }^{5}$ (D) \\ ${ }^{1}$ Department of Gastroenterology, Ankara Atatürk Training and Research Hospital, Ankara, Turkey \\ 2Department of Clinical Biochemistry, Ankara Atatürk Training and Research Hospital, Ankara, Turkey \\ ${ }^{3}$ Department of Gastroenterology, Yıldırım Beyazıt University School of Medicine, Ankara, Turkey \\ ${ }^{4}$ Department of Gastroenterology, Çorlu State Hospital, Tekirdağ, Turkey \\ ${ }^{5}$ Department of Clinical Biochemistry, Yıldırım Beyazıt University School of Medicine, Ankara, Turkey
}

Cite this article as: Köseoğlu H, Alışık M, Başaran M, et al. Dynamic thiol/disulphide homeostasis in acute pancreatitis. Turk J Gastroenterol 2018; 29: 348-53.

\begin{abstract}
Background/Aims: The dynamic thiol/disulfide homeostasis plays pivotal roles in many physiological mechanisms in an organism. We aimed to investigate whether dynamic thiol/disulfide homeostasis changes among patients with acute pancreatitis.

Materials and Methods: This prospective trial contained 45 patients with acute pancreatitis and 45 sex-and age-matched healthy volunteers as control group. Thiol/disulfide homeostasis parameters were measured by a novel and automated assay, and detected results were compared between the two groups.

Results: Disulfide/total thiol percent ratio and disulfide/native thiol percent ratios were significantly higher in acute pancreatitis group; besides the native thiol, total thiol levels and native thiol/total thiol percent ratios were significantly lower (for all $p<0.001$ ).

Conclusion: The thiol/disulfide homeostasis is impaired in acute pancreatitis with a shift toward the oxidative status, and this deficiency might be a pathogenic factor in acute pancreatitis. The correction of this thiol/disulfide imbalance may be a new target in the management of acute pancreatitis.
\end{abstract}

Keywords: Acute pancreatitis, disulphide bond, oxidative stress, thiol

\section{INTRODUCTION}

Acute pancreatitis (AP), a frequently seen disease in clinical practice, annually affects around 80 per 100,000 individuals (1). Severe AP has been defined as AP with persistent organ failure and has a great morbidity and mortality $(2,3)$. A recently defined group is moderately severe $A P$, which consists of patients who have AP with transient organ failure or local or systemic complications without persistent organ failure $(2,4)$. Mild AP, which is defined as the absence of both organ failure and complications, is the mostly encountered form of AP in clinical practice (2).

A sulfur atom and a hydrogen atom come together to form a sulfhydryl group. Thiols are organic compounds with such sulfhydryl groups attached to a carbon atom (5). When oxidation occurs, they form a covalent bond named disulfide bonds (also called as SS-bond). In oxidative stress (OS) status, the oxidation of cysteine (Cys) residues leads to the reversible formation of mixed disulfides. These disulfide bonds may be reduced to thiol groups, so that a dynamic homeostasis could be ensured $(6,7)$. This thiol/disulfide homeostasis plays important vital roles in detoxification, antioxidant protection, regulation of enzymatic activity and transcription factors, apoptosis, and cellular signal mechanisms $(8,9)$.

Since 1979, just one side of the double-sided balance could be measured by a method defined by Ellman et al. (10). A novel, relatively cheap, readily available, and automated assay has been recently described by Erel and Neselioglu, which measures the serum total thiol and native thiol levels and calculates disulfide concentrations, so that the dynamic thiol/disulfide homeostasis can be determined (7).

The present study proposed to investigate thiol/disulfide homeostasis parameters, a kind of homeostasis which 
takes place in the maintenance of many physiological mechanisms essential for the organism, in patients with AP. There is no other report in the present literature investigating the association between dynamic thiol/disulfide homeostasis and AP.

\section{MATERIALS AND METHODS}

This prospective study included 45 patients with AP and 45 sex-and age-matched healthy volunteers with symptoms suggesting functional dyspepsia and normal serum amylase and lipase levels as control group. The diagnosis of AP was defined as the presence of two of the three requested diagnostic criteria: (i) abdominal pain compatible with AP, (ii) serum lipase or amylase levels higher than three times the upper limit of normal, and (iii) characteristic findings from abdominal imaging (11). The severity of AP was assessed with the aid of the criteria proposed by the International Atlanta Symposium on Acute Pancreatitis (2). Patients with chronic pancreatitis, malignant diseases, renal failure, liver failure, heart failure, myocardial infarction, rheumatic diseases, and immunosuppressive disorders were excluded because these conditions may have significant effects on thiol/disulfide homeostasis.

Age, gender, etiology of AP, white blood count, serum C-reactive protein (CRP), amylase and lipase levels, the Ranson score, the Balthazar score, the Acute Physiology and Chronic Health Enquiry (APACHE II) score, and the duration of hospital stay were recorded during the hospital stay.

Fasting venous blood samples were obtained from patients with AP upon admission to the gastroenterology clinic and from controls and were centrifuged at 1200x $\mathrm{g}$ for $15 \mathrm{~min}$. Thereafter, serum samples were stored at $-80^{\circ} \mathrm{C}$ until the investigation time. Serum native thiol and total thiol levels were measured with novel and fully automated tests described by Erel and Neselioglu. After serum extraction, the test takes about 12 min to measure these values with the automated system. Disulfide concentrations were calculated as the half of the difference between levels of the total thiol and native thiol. Then, disulfide/total thiol percent ratio, disulfide/native thiol percent ratio, and native thiol/total thiol percent ratio were calculated (7).

Written informed consent was obtained according to a protocol approved by local institutional ethical committee.

Statistical Package for Social Sciences 17.0 (SPSS Inc.; Chicago, IL, USA) software was used for statistical analy- ses. Kolmogorov-Smirnov test was used to determine the normal distribution of variables. Independent samples t-test was used to determine the differences in variables between AP and control groups, and Spearman's correlation analysis was used to identify the correlation between clinical and biochemical prognostic factors, length of hospital stay, and thiol/disulfide homeostasis parameters. Kruskal-Wallis test was used to compare thiol/disulfide homeostasis parameters according to the etiology of AP. $p$ values of less than 0.05 were considered as significant.

\section{RESULTS}

This report included 45 patients with AP and 45 healthy volunteers. The groups were similar according to age and gender (Table 1). The etiology of AP was gallstones in 24 patients $(53.3 \%)$, hypertriglyceridemia in six patients $(13.3 \%)$, alcohol use in three patients $(6.7 \%)$, and drug use in three patients $(6.7 \%)$. In nine patients $(20 \%)$, the predisposing factor for AP could not be detected by the available diagnostic techniques. The mean duration of hospital stay was 7.7 days with no in-hospital mortality. The mean total Ranson score was $1.3(0-5)$, APACHE II score 2.3 (0-9), and the mean Balthazar score was 1.9 (07). Among the patients with AP, 40 had mild AP, four had moderately severe AP, and one patient had severe AP. The etiology of the patient with severe AP was drug use. Three patients with idiopathic pancreatitis and one patient with biliary pancreatitis had moderately severe AP.

Disulfide/total thiol percent ratios and disulfide/native thiol percent ratios were significantly higher in AP group, whereas the total thiol level, native thiol level, and native thiol/total thiol percent ratios were significantly lower (Figure 1 and Table 1). Mean disulfide level was higher in the AP group, with a non-significant difference (Table 1). The results showed no statistically significant difference in thiol/disulfide homeostasis parameters according to the etiology of AP (Table 2). In patient whose AP etiology was alcohol or drug use, the disulfide/total thiol percent ratios and disulfide/native thiol percent ratios were higher, and the total thiol level, native thiol level, and native thiol/total thiol percent ratios were lower than the other etiologic factors, but the difference showed no significant difference. The thiol/disulfide homeostasis parameters were not statistically significantly different according to gender and age (data not shown).

On correlation analysis, thiol/disulfide homeostasis components were not correlated with the hospital stay duration, APACHE II score, Ranson score, Balthazar score, amylase, lipase, CRP, white blood count, and age. 


\section{DISCUSSION}

Our study showed that disulfide/total thiol percent ratios and disulfide/native thiol percent ratios were significantly higher in patients with $A P$, whereas the total thiol level, native thiol level, and native thiol/total thiol percent ratios were lower. Dynamic thiol/disulfide homeostasis status plays critical and vital roles in detoxification, antioxidant protection, apoptosis, signal transduction, regulation of enzymatic activity, and transcription factors and cellular signaling mechanisms $(8,9)$. Thiols are considered as essential antioxidant buffers because they are in continuous interaction with nearly all physiological oxidants (12). Thiol protein groups are

Table 1. Sociodemographic features and thiol/disulfide homeostasis parameters in both groups

\begin{tabular}{lcccc}
\hline & $\begin{array}{c}\text { Control } \\
\text { group } \\
(\mathrm{n}: 45)\end{array}$ & $\begin{array}{c}\text { Acute } \\
\text { pancreatitis } \\
\text { group (n:45) }\end{array}$ & $\mathrm{p}$ \\
\hline Age & $49.62 \pm 10.92$ & $52.76 \pm 17.68$ & 0.31 \\
Male $(\mathrm{n})$ & $20(44.4 \%)$ & $20(44.4 \%)$ & 1 \\
Native thiol; $\mu \mathrm{mol} / \mathrm{L}$ & $435.48 \pm 81.63$ & $294.74 \pm 107.54$ & $<0.001$ \\
Total thiol; $\mu \mathrm{mol} / \mathrm{L}$ & $483.66 \pm 78.57$ & $345.98 \pm 100.75$ & $<0.001$ \\
Disulphide; $\mu \mathrm{mol} / \mathrm{L}$ & $24.21 \pm 6.19$ & $25.81 \pm 12.65$ & 0.23 \\
$\begin{array}{l}\text { Disulphide/total thiol } \\
\text { percent ratios }(\%)\end{array}$ & $5.17 \pm 1.69$ & $8.21 \pm 4.77$ & $<0.001$ \\
$\begin{array}{l}\text { Disulphide/native thiol } \\
\text { percent ratios }(\%)\end{array}$ & $5.85 \pm 10.66$ & $10.66 \pm 7.91$ & $<0.001$ \\
Native thiol/total thiol & $89.70 \pm 3.37$ & $83.72 \pm 9.44$ & $<0.001$ \\
percent $(\%)$ & & & \\
\hline
\end{tabular}

The values are expressed as mean \pm standard deviation for continuous variables and number (percent) for categorical variables important antioxidants, which constitute for $52.9 \%$ of the total serum antioxidant capacity in healthy individuals (13). Erel and Neselioglu described a novel automated test determining dynamic thiol/disulfide homeostasis. With this method, serum total thiol and native thiol levels are measured and disulfide concentrations are calculated with these levels (7). The results of the thiol/ disulfide homeostasis tests show the balance shift toward the thiol or disulfide side. With this newly defined measurement method, deficient thiol/disulfide homeostasis has been found in various clinical situations, such as hyperemesis gravidarum, acute myocardial infarction, non-ST elevation myocardial infarction, and type I diabetes mellitus (12,14-16). Reduced glutathione is the main non-protein thiol in mammalian cells and is an important antioxidant (17). Its depletion in the pancreatic tissue has been shown in animal models of AP (17-19). These studies used pancreatic tissue for thiol measurements, whereas our research is the first study measuring thiol/disulfide homeostasis parameters in serum with an easy and a fully automated way in patients with AP. Moreno et al. (20) evaluated low-molecular-weight thiols and disulfides in an animal model with AP. In this study, disulfide stress was considered as a specific type of OS associated with mixed disulfides, particularly protein cysteinylation, and oxidation of thiols such as gamma-glutamylcysteine, cysteine, and homocysteine. But glutathione oxidation or changes in protein glutathionylation were not detected. In this study, reduced glutathione level was also decreased in the pancreatic tissue of rats with AP (20). Disulfide/total thiol percent ratios and disulfide/native thiol percent ratios were significantly higher in patients with AP, whereas the total and native thiol levels and native thiol/total thiol percent ratio were significantly lower. These changes indicate

Table2. Thiol/disulfide homeostasis parameters due to acute pancreatitis etiology

\begin{tabular}{|c|c|c|c|c|c|}
\hline & $\begin{array}{c}\text { Gallstone } \\
(\mathrm{n}: 24)\end{array}$ & $\begin{array}{l}\text { Hypertriglyceridemia } \\
\qquad(\mathrm{n}: 6)\end{array}$ & $\begin{array}{c}\text { Alcohol } \\
(\mathrm{n}: 3)\end{array}$ & $\begin{array}{l}\text { Drug use } \\
\text { (n:3) }\end{array}$ & $\begin{array}{l}\text { Idiopathic } \\
(\mathrm{n}: 9)\end{array}$ \\
\hline Native thiol; $\mu \mathrm{mol} / \mathrm{L}$ & 276.50 & 278 & 217 & 256 & 351 \\
\hline Total thiol; $\mu \mathrm{mol} / \mathrm{L}$ & 329.50 & 335.50 & 306 & 303 & 374 \\
\hline Disulphide; $\mu \mathrm{mol} / \mathrm{L}$ & 25.38 & 19.23 & 45 & 32 & 24 \\
\hline Disulphide/total thiol percent ratios (\%) & 7.65 & 5.33 & 12.96 & 9.58 & 7.98 \\
\hline Disulphide/native thiol percent ratios (\%) & 9.05 & 6.18 & 17.39 & 11.85 & 6.88 \\
\hline Native thiol/total thiol percent ratios (\%) & 84.72 & 89.52 & 74.54 & 80.84 & 86.24 \\
\hline
\end{tabular}


a

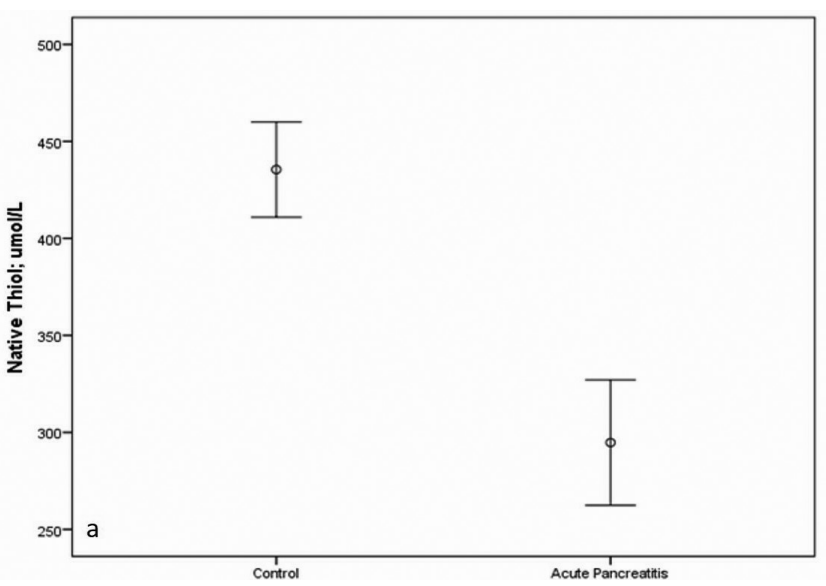

C

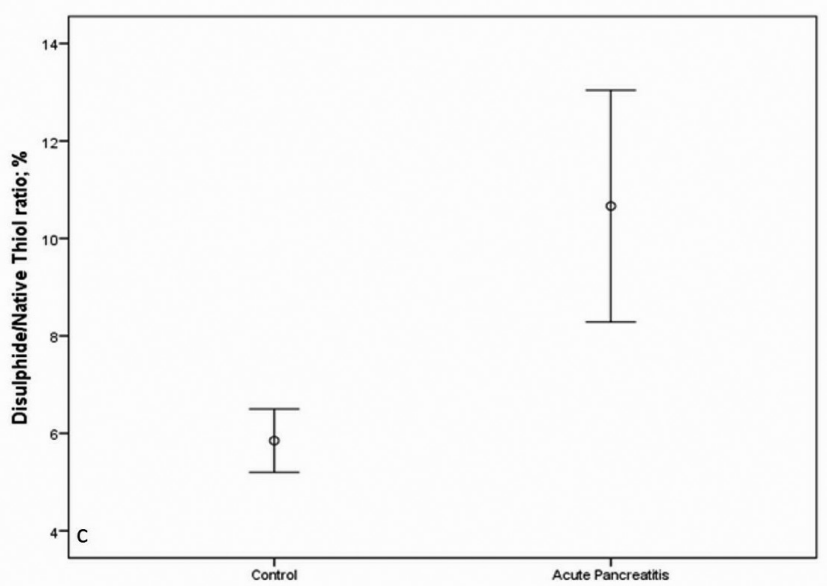

b

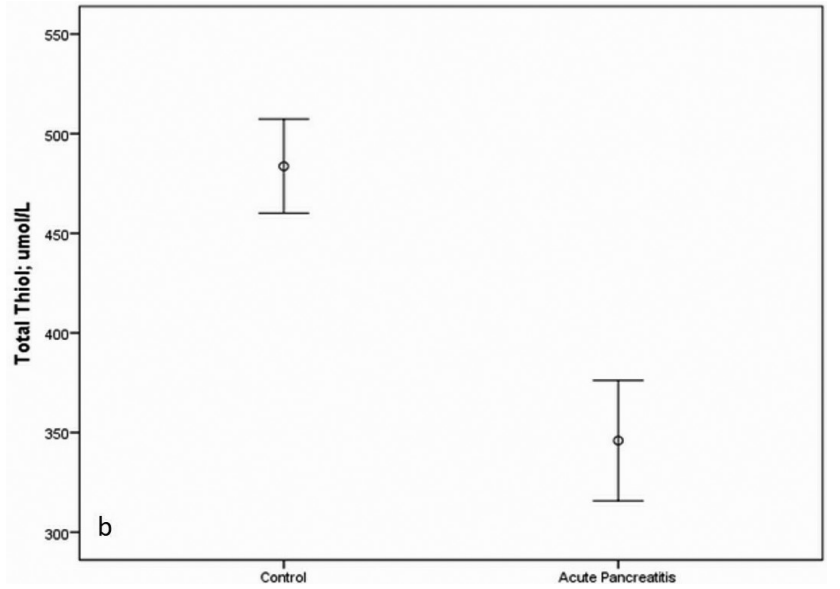

d

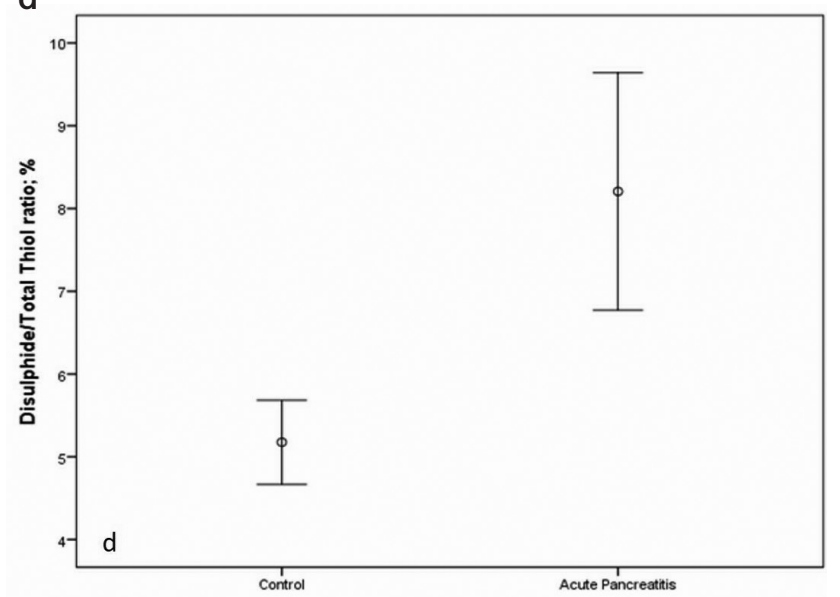

Figure 1. a-d. Native thiol (a), total thiol (b), disulfide/native thiol ratio (c), and disulfide/total thiol ratios (d) among the control and AP groups

that the thiol/disulfide redox balance shifted to the disulfide bond side in AP. To our best knowledge, this is the first study demonstrating with an easy method that dynamic thiol/disulfide homeostasis changes and the balance changes to the side of disulfide bond formation or namely to the oxidative side.

Although our study is the first study evaluating dynamic thiol/disulfide homeostasis parameters with an easy method, similar results were obtained from previous studies with different procedures reflecting OS. The plasma total antioxidant status levels were reduced in two studies and plasma total oxidative status was increased $(21,22)$. In this mentioned study, Dur et al. (21) found that lymphocyte oxidative DNA damage, which is a marker for OS, was also higher among patients with AP than that among control group. A recently published study also showed an increase in OS and a decrease in antioxidant levels (23). Bopanna et al. (23) showed that 4-hydroxynonenol levels increased in patients with idiopathic recurrent $A P$, reflecting $O S$. The antioxidant levels were measured by ferric reducing the ability of plasma, and a significant reduction was obtained in patients with idiopathic recurrent AP. Ischemia-modified albumin, protein carbonyls, and superoxide dismutase are other examples of markers for OS, which were found to be elevated among AP in different trials $(22,24,25)$. When our results are considered with these findings, our study implies the possible role of OS in the pathogenesis of AP, but these results may only reflect altered oxidative status. A recently published meta-analysis revealed possible benefit of antioxidant therapy for AP, and this may also be an evidence that the depletion of antioxidants take place in the pathogenesis of AP (26). 
No correlation was observed between CRP level, length of hospital stay, APACHE II score, Ranson score, Balthazar score, and thiol/disulfide homeostasis parameters. Most of our patients had mild AP, and we could not compare mild and severe AP. Owing to these reasons, we cannot advice at this moment if thiol/disulfide homeostasis components as prognostic predictors in AP.

Our findings suggest that the deficiency of thiol/disulfide homeostasis may play a role in the pathogenesis of AP. The supplementation with thiol compounds such as $\mathrm{N}$-acetyl cystein (NAC) may be a target for the therapy of AP. NAC is a free radical scavenger, which stimulates glutathione synthesis (27). To date, animal studies demonstrated that NAC had reduced the severity of $\operatorname{AP}(27,28)$. It was also found that mixed antioxidant supplementation including NAC might decrease the duration of hospital stay and complication rates in patients suffering from AP (29). NAC treatment failed to prevent patients from developing post-endoscopic retrograde cholangiopancreatography pancreatitis (PEP) in two studies, but in one study, a higher dose of NAC reduced the probability of developing PEP (30-32). These conflicting results may be due to the different doses of NAC used in these trials. Another explanation is that there may be a point of no return, and administration of therapy after that point may be inadequate to prevent AP progression, and one can speculate that early admission of NAC may be essential for the treatment of AP. Human studies investigating NAC in AP treatment are not widely available in the literature, but promising results about NAC in AP treatment were found in a study with a relatively low study population (29). We can speculate that easily and rapidly measurable serum thiol/disulfide parameters may be used to define the optimal dose for NAC or other antioxidant therapy for AP in future studies.

We have to mention some limitations of our study. First of all, the low number of the study population is our major limitation. Although this number was low, statistically significant results were achieved. Among the AP group, only one patient had severe AP and four patients had moderately severe AP. If we had more patients with severe $A P$, we could compare mild $A P$ with severe $A P$ and could evaluate thiol/disulfide parameters as prognosis factors. Future studies comparing mild with severe AP can help determine thiol/disulfide parameters as prognosis factors. Another limitation of our study is that some laboratory parameters were absent in the control group, and we could not compare simple laboratory parameters between groups.
In conclusion, dynamic thiol/disulfide homeostasis is altered in AP. The deficiency of thiol/disulfide homeostasis may be involved in the pathogenesis of AP, and the supplementation with thiol compounds may become a therapeutic option of AP in future studies despite some conflicting results.

Ethics Committee Approval: Ethics committee approval was received for this study from the Local Institutional Ethical Committee.

Informed Consent: Written informed consent was obtained from all the patients who participated in this study.

Peer-review: Externally peer-reviewed.

Author Contributions: Concept - H.K., O.E.; Design - Ö.T.Y., M.B.; Supervision - M.A., Ö.E., M.T.; Resources - O.E., Ö.E., M.A.; Materials - M.A., Ö.T.Y., T.S.; Data Collection and/or Processing - H.K., Ö.T.Y.; Analysis and/or Interpretation - H.K., T.S., O.E.; Literature Search H.K., M.T., M.B.; Writing Manuscript - H.K., Ö.T.Y., T.S.; Critical Review - T.S., M.T., O.E., Ö.E.

Conflict of Interest: The authors have no conflict of interest to declare.

Financial Disclosure: The authors declared that this study has received no financial support.

\section{REFERENCES}

1. Hamada $T$, Yasunaga $H$, Nakai $Y$, et al. Japanese severity score for acute pancreatitis well predicts in-hospital mortality: a nationwide survey of 17,901 cases. J Gastroenterol 2013; 48: 1384-91. [CrossRef] 2. Banks PA, Bollen TL, Dervenis C, et al. Classification of acute pancreatitis - 2012: revision of the Atlanta classification and definitions by international consensus. Gut 2013; 62: 102-11. [CrossRef]

3. Chen Y, Ke L, Tong Z, Li W, Li J. Association between severity and the determinant-based classification, Atlanta 2012 and Atlanta 1992, in acute pancreatitis: a clinical retrospective study. Medicine (Baltimore) 2015; 94: e638. [CrossRef]

4. Vege SS, Gardner TB, Chari ST, et al. Low mortality and high morbidity in severe acute pancreatitis without organ failure: a case for revising the Atlanta classification to include "moderately severe acute pancreatitis". Am J Gastroenterol 2009; 104: 710-5. [CrossRef] 5. Sen CK, Packer L. Thiol homeostasis and supplements in physical exercise. Am J Clin Nutr 2000; 72(2 Suppl.): 653S-69S. [CrossRef] 6 . Jones DP, Liang Y. Measuring the poise of thiol/disulfide couples in vivo. Free Radic Biol Med 2009; 47: 1329-38. [CrossRef]

7. Erel $O$, Neselioglu S. A novel and automated assay for thiol/disulphide homeostasis. Clin Biochem 2014; 47: 326-32. [CrossRef] 8. Biswas S, Chida AS, Rahman I. Redox modifications of protein-thiols: emerging roles in cell signaling. Biochem Pharmacol 2006; 71: 551-64. [CrossRef]

9. Circu ML, Aw TY. Reactive oxygen species, cellular redox systems, and apoptosis. Free Radic Biol Med 2010; 48: 749-62. [CrossRef]

10. Ellman G, Lysko H. A precise method for the determination of whole blood and plasma sulfhydryl groups. Anal Biochem 1979; 93: 98-102. [CrossRef]

11. Tenner S, Baillie J, DeWitt J, Vege SS; American College of Gastroenterology. American College of Gastroenterology guideline: 
management of acute pancreatitis. Am J Gastroenterol 2013; 108 : 1400-15. [CrossRef]

12. Ergin M, Cendek BD, Neselioglu S, Avsar AF, Erel O. Dynamic thiol-disulfide homeostasis in hyperemesis gravidarum. J Perinatol 2015; 35: 789-92. [CrossRef]

13. Erel O. A novel automated direct measurement method for total antioxidant capacity using a new generation, more stable ABTS radical cation. Clin Biochem 2004; 37: 277-85. [CrossRef]

14. Kundi $H$, Ates I, Kiziltunc $E$, et al. A novel oxidative stress marker in acute myocardial infarction; thiol/disulphide homeostasis. Am J Emerg Med 2015; 33: 1567-71. [CrossRef]

15. Kundi $\mathrm{H}$, Erel Ö, Balun A, et al. Association of thiol/disulfide ratio with syntax score in patients with NSTEMI. Scand Cardiovasc J 2015; 49: 95-100. [CrossRef]

16. Ates I, Kaplan M, Yuksel M, et al. Determination of thiol/disulphide homeostasis in type 1 diabetes mellitus and the factors associated with thiol oxidation. Endocrine 2016; 51: 47-51. [CrossRef]

17. Pérez S, Pereda J, Sabater L, Sastre J. Redox signaling in acute pancreatitis. Redox Biol 2015; 5: 1-14. [CrossRef]

18. Dabrowski A, Chwiecko M. Oxygen radicals mediate depletion of pancreatic sulfliydryl compounds in rats with cerulein-induced acute pancreatitis. Digestion 1990: 47: 15-9. [CrossRef]

19. Schoenberg MH, Büchler M, Gaspar M, et al. Oxygen free radicals in acute pancreatitis of the rat. Gut 1990; 31: 1138-43. [CrossRef]

20. Moreno ML, Escobar J, Izquierdo-Álvarez A, et al. Disulfide stress: a novel type of oxidative stress in acute pancreatitis. Free Radic Biol Med 2014; 70: 265-77. [CrossRef]

21. Dur A, Kocaman O, Koçyiğit A, et al. Oxidative status and lymphocyte DNA damage in patients with acute pancreatitis and its relationship with severity of acute pancreatitis. Turk J Gastroenterol 2016; 21: 68-72. [CrossRef]

22. Baser H, Can U, Karasoy D, et al. Evaluation of oxidant/anti-oxidants status in patients with mild acute -pancreatitis. Acta Gastroenterol Belg 2016; 79: 23-8.

23. Bopanna S, Nayak B, Prakash S, Shalimar, Mahapatra SJ, Garg PK. Increased oxidative stress and deficient antioxidant levels may be involved in the pathogenesis of idiopathic recurrent acute pancreatitis. Pancreatology 2017; 17: 529-33. [CrossRef]

24. Winterbourn CC, Bonham MJ, Buss H, Abu-Zidan FM, Windsor JA. Elevated protein carbonyls as plasma markers of oxidative stress in acute pancreatitis. Pancreatology 2003; 3: 375-82. [CrossRef]

25. Thareja S, Bhardwaj P, Sateesh J, Saraya A. Variations in the levels of oxidative stress and antioxidants during early acute pancreatitis. Trop Gastroenterol 2009; 30: 26-31.

26. Jeurnink SM, Nijs MM, Prins HA, Greving JP, Siersema PD. Antioxidants as a treatment for acute pancreatitis: A meta-analysis. Pancreatology 2015; 15: 203-8. [CrossRef]

27. Du BQ, Yang YM, Chen YH, Liu XB, Mai G. N-acetylcysteine improves pancreatic microcirculation and alleviates the severity of acute necrotizing pancreatitis. Gut Liver 2013; 7: 357-62. [CrossRef] 28. Onur E, Paksoy M, Baca B, Akoglu H. Hyperbaric oxygen and $\mathrm{N}$-acetylcysteine treatment in L-arginine-induced acute pancreatitis in rats. J Invest Surg 2012; 25: 20-8. [CrossRef]

29. Sateesh J, Bhardwaj P, Singh N, Saraya A. Effect of antioxidant therapy on hospital stay and complications in patients with early acute pancreatitis: a randomised controlled trial. Trop Gastroenterol 2009; 30: 201-6.

30. Katsinelos P, Kountouras J, Paroutoglou G, Beltsis A, Mimidis K, Zavos $C$. Intravenous $N$-acetylcysteine does not prevent post-ERCP pancreatitis. Gastointest Endosc 2005; 62: 105-11. [CrossRef] 31. Milewski J, Rydzewska G, Degowska M, Kierzkiewicz M, Rydzewski A. N-acetylcysteine does not prevent post-endoscopic retrograde cholangiopancreatography hyperamylasemia and acute pancreatitis. World J Gastroenterol 2006; 12: 3751-5. [CrossRef]

32. Alavi Nejad P, Hajiani E, Hashemi J, Masjedizadeh AR, Shayesteh AA, Sebghatollahi V. Evaluation of N-acetyl Cysteine for the Prevention of Post-endoscopic Retrograde Cholangiopancreatography Pancreatitis: A Prospective Double Blind Randomized Pilot Study. Middle East J Dig Dis 2013; 5: 17-21. 